\title{
Early dynamical evolution of young substructured clusters
}

\author{
Julien Dorval and Christian Boily \\ Observatoire Astronomique, Université de Strasbourg, 11 rue de l'Université, 67000 \\ Strasbourg, France \\ email: julien.dorval@astro.unistra.fr \\ email: christian.boily@astro.unistra.fr
}

\begin{abstract}
Stellar clusters form with a high level of substructure, inherited from the molecular cloud and the star formation process. Evidence from observations and simulations also indicate the stars in such young clusters form a subvirial system. The subsequent dynamical evolution can cause important mass loss, ejecting a large part of the birth population in the field. It can also imprint the stellar population and still be inferred from observations of evolved clusters. Nbody simulations allow a better understanding of these early twists and turns, given realistic initial conditions. Nowadays, substructured, clumpy young clusters are usually obtained through pseudo-fractal growth and velocity inheritance. We introduce a new way to create clumpy initial conditions through a "Hubble expansion" which naturally produces self consistent clumps, velocity-wise. In depth analysis of the resulting clumps shows consistency with hydrodynamical simulations of young star clusters. We use these initial conditions to investigate the dynamical evolution of young subvirial clusters. We find the collapse to be soft, with hierarchical merging leading to a high level of mass segregation. The subsequent evolution is less pronounced than the equilibrium achieved from a cold collapse formation scenario.
\end{abstract}

Keywords. Star cluster, fragmentation, Nbody simulations

\section{Introduction}

Observed young stellar cluster often display a complex, clumpy structure (Kuhn et al. 2014). This is thought to originate from the localized star formation in the turbulent molecular cloud. These substructures are bound to be erased through dynamical evolution and relaxation as older clusters appear much smoother and spherical (Andre et al. 2007). This dynamical evolution comes off as a crucial phase in the life of the cluster, setting the longer lived, global structure and ejecting a part of the birth population. A good understanding of this process is of major importance in a study of the life cycle of star clusters. Nbody simulations allow to gain insight into the dynamical processes at play in this problem.

However, a critical aspect of nbody simulations are initial conditions. Older, spherical clusters can be easily reproduced by sampling well known King or Plummer distributions. To generate young substructured clusters constitutes however a real challenge. Several solutions have been adopted in the past to create clumpy initial conditions. For example, one can create several plummer clumps and scatter them in space, effectively creating a clumpy cluster, see McMillan et al. (2007). Though simple and straightforward, this approach forces an unjustified internal spatial distribution on clumps. Another widely used method is the fractal growth method from Goodwin et al. (2004). Through recursive probabilistic spawning of "children", one can grow a pseudo-fractal star cluster. The outcome of this process is visually very realistic and make convincing clumpy young clusters. However, the velocity distribution, though not absurd, is completely artificial. 

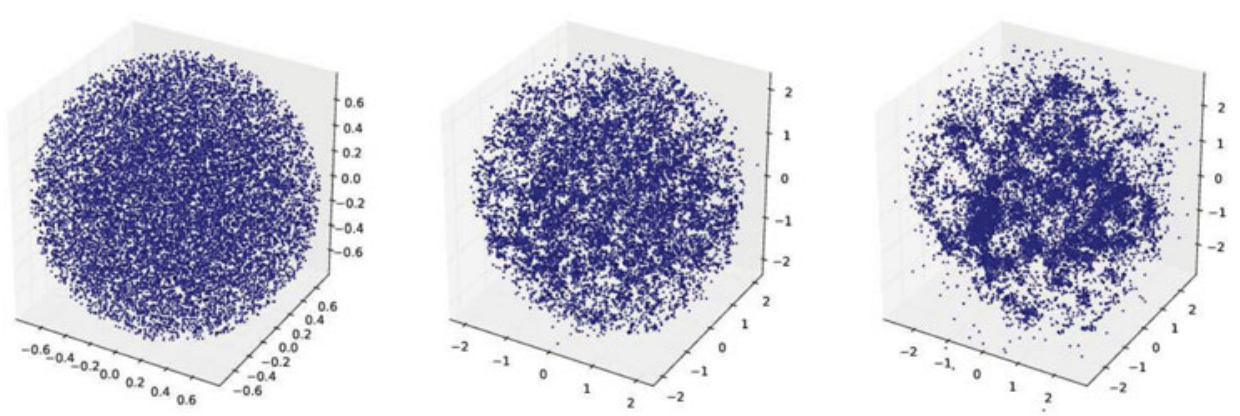

Figure 1. Progressive fragmentation through the Hubble expansion. Axis were rescaled to preserve the model's appearance, even though the radius increases. Left panel shows the initial uniform sphere, middle panel an intermediate step, slightly fragmented with a drastically slowed down expansion, right is the final stage, the expansion has stopped and the fragmentation is fully developed.

When integrated, such a system will undergo immediate relaxation within the clumps, which can be an issue.

We introduce here a new method to create self consistent clumpy initial conditions. We start with an uniform sphere model, in which we spawn the requested number of stars. We then attribute velocities according to a Hubble law $\mathbf{v}=H \mathbf{r}, H$ being a "Hubblelike" constant, tuning the rate of expansion. When using the Hénon unit system (Hénon 1973), $H$ must be lower than 1.4 to keep the system bound. We then feed this system to a collisional Nbody integrator, which is NBODY6 in our case. The system expands until it reaches the turning point, at which the expansion stops and the system is about to collapse. We stop the integration at this moment. During the expansion, density fluctuations in the initial uniform sphere grow, which leads to fragmentation. The resulting system is substructured in a fully self-consistent fashion. The clumps $\dagger$ are spontaneous and have self-consistent velocity distributions. When integrated, the system won't be undergoing local initial relaxation like fractal models, as the stars already know of each other's gravitational pull. By construction, the system is subvirial and will collapse when integrated.

\section{Clump analysis}

To study the resulting substructured system, one first needs to isolate the clumps. To do so we used the Minimum Spanning Tree cut off method, from Maschberger et al. (2010). The idea is to first build the three-dimensionnal Minimum Spanning Tree of the system, which is the shortest branching path to link all the points. Then a cut-off length is chosen, and all edges in the tree longer than this length are deleted. The resulting isolated subtrees are considered clumps if they contain more than 12 particles. The cutoff length is the only free parameter of the method. We developped a method to remove this free parameter, which we detail in Dorval et al. (2015, MN submitted).

The first aspect of clumps which is of interest to us is the mass segregation. A widelyused method to assess mass segregation is the mass segregation ratio (Allison et al. 2009). However, such a method is not adapted to very small systems, of the order of 20 members. We chose instead to implement the radial ranking method from Maschberger et al. (2010). For each clump, the distances from stars to the geometrical center of the clumps are computed, then sorted. The positions of the first, second, and third

$\dagger$ In this paper, we use "clump" to mean, loosely, stellar associations. 


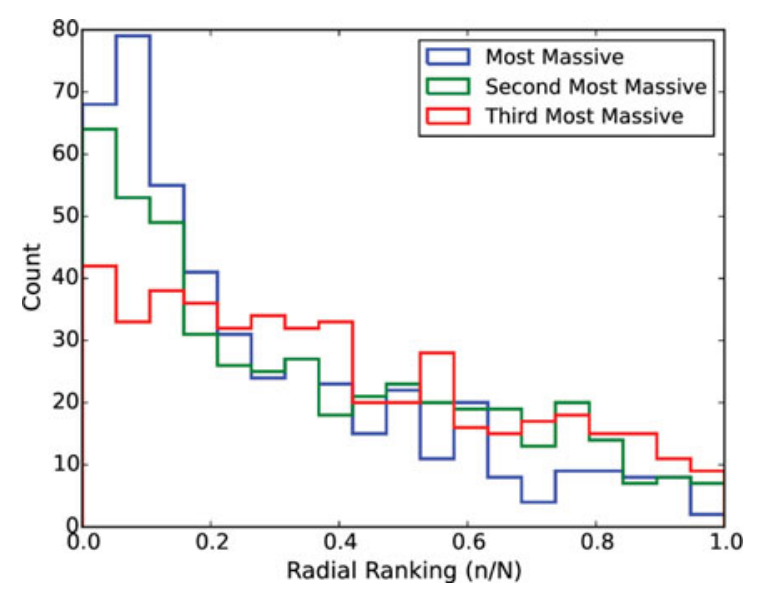

Figure 2. Radial ranking of first, second and third most massive stars for all clumps in a 40,000 particles Hubble fragmented model.

most massive stars in this ranking are converted into a 0 to 1 factor. Plotting the three histograms (first, second and third most massive) once the process is applied to all clumps allows to assess the trend towards mass segregation at a glance. In a non-segregated system, these histograms are flat on average. Figure 1 shows the results for a fragmented Hubble model. The histograms clearly show a mass segregation in the clumps. The results show trends similar to those seen in the analysis of Maschberger et al. (2010) applied to stellar cores formed hydrodynamically.

The origin of this primordial segregation was explained by Maschberger et al. (2010) through the clump formation process. In their hydrodynamical simulations, the clumps form around the most massive stars, which act as gravitationnal seeds and attract low mass stars. The same phenomenon is at play during the Hubble fragmentation. To confirm this, we turn to the clump stellar content. On figure 2, we show several mass functions in our fragmented model. In green is the global stellar mass function of the cluster, which follows a Salpeter law from 0.35 top $20 M_{\odot}$. In red is the mass function of all stars registered as part of a clump. Finally, in blue is the theoretical Salpeter distribution for the same number of stars than what was found in clumps, that is to say the expected shape of the red distribution. Clumps are clearly top-heavy, most of the massive stars in the system end up in a clump, resulting in a shallower power-law $(\alpha=2.15)$ for members. This is consistent with them acting as fragmentation seeds.

These two results, mass segregation and top-heavy clumps, confirm that massive stars act as seeds for clump formation. Heavier stars form clumps around them, thus both preferentially belonging to a clump and sitting at the center.

\section{Dynamical evolution}

Once the fragmented system is obtained, we can let it evolve further to investigate the fate of young substructured and subvirial clusters. We take a 15000 particle system, drawn from a Salpeter stellar mass function from 0.35 to $20 M_{\odot}$. This gives a mean stellar mass of $1 M_{\odot}$ and a total mass of $15000 M_{\odot}$. The Nbody calculations to obtain the system were done in Hénon units (Hénon 1973). We pick scales adapted to real systems: for a half-mass radius of $1 \mathrm{pc}$, the resulting stellar density $\rho=M /\left(8 \pi / 3 r_{h}^{3}\right) \simeq 10^{3} M_{\odot} / p c^{3}$ is consistent with several observations. We let the system evolve for 40 Henon time units, translating to about 1 Myr. For comparison purposes, we performed similar computations 


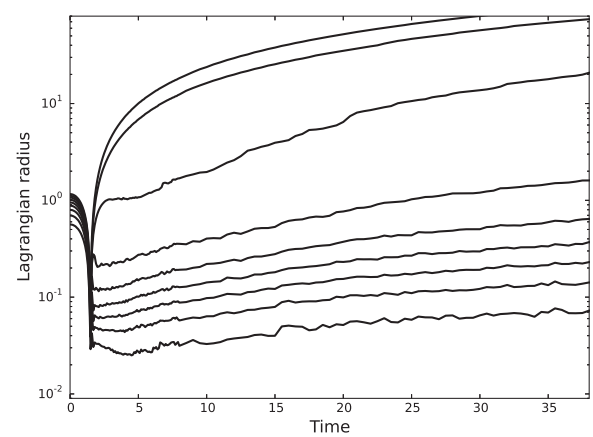

(a) Cold uniform collapse

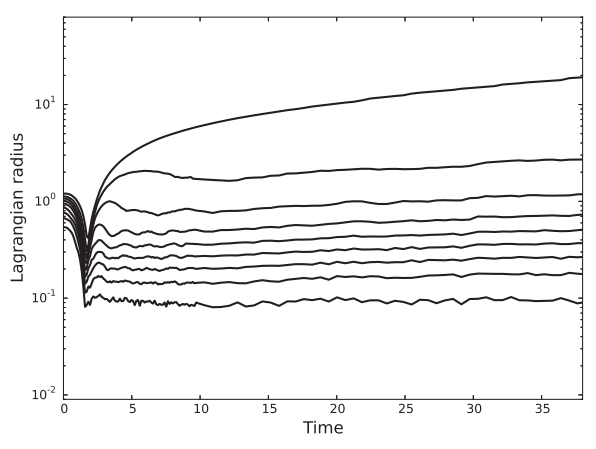

(b) Hubble collapse

Figure 3. Ten percentile lagrangian radii for both types of collapse. Time and radii are in Henon units.

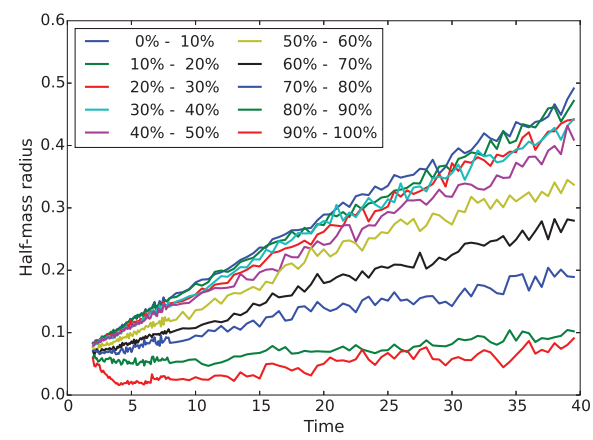

(a) Cold uniform collapse

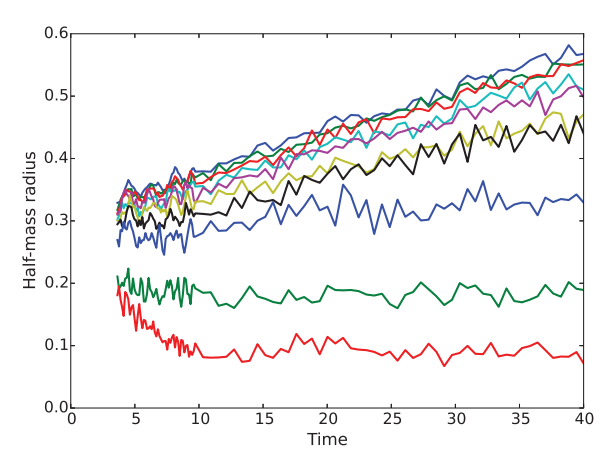

(b) Hubble collapse

Figure 4. Half-mass radii for ten mass-sorted subpopulations. Time and radii are in Henon units.

with a cold uniform sphere model of same mass and size. On figure 3 we show the ten percentile Lagrangian radii for the Hubble fragmented system (3a) and cold uniform (3b). The uniform collapse appears deeper and has a stronger "bounce", as twice as many stars are ejected. From a shallower post-collapse state, the Hubble model displays a slower evolution. The overall structure of the system does not change appreciably from a time $t \approx 5$ units until the end of the run, whereas the cold uniform system is clearly expanding throughout the simulation.

We also wish to investigate the mass segregation in the evolution of our system, as a follow up to the study on the individual clumps. To look at the overall system, we chose another indicator of mass segregation. We take inspiration from Caputo et al. (2014) and use their method. The masses are sorted by increasing values, then subdivided into ten equal-mass bins. This means that the first bin contains the least massive stars, and holds the largest number of stars. The number of stars in each bin decreases as we shift to the following bins, since their mean mass increases, and so on until we have binned all the stars. The half-mass radius computed for each bin is then plotted as function of time. In this way the mass segregation un-folds over time : if the stars were not segregated by mass, all half-mass radii would overlap. We show on figure 4 the outcome of the analysis for both uniform and Hubble models. We removed stars that were ejected after collapse, thus no data is available before that. Both uniform and Hubble models develop mass segregation over time, as the different populations separate themselves spatially. However, the Hubble 
model has a head-start in mass segregation as the different half-mass radii are already well separated immediately after the collapse. This is not surprising as clumps were found to be mass segregated prior to collapse, and inheritance of segregation during mergers is a known result (see McMillan et al. 2007). The denser configuration after the collapse in the cold uniform model induce stronger two-body interaction and segregation increases faster than for the Hubble configuration. In the end, after evolving for the same amount of time, the uniform model appears more segregated than the Hubble model.

Whether in regards to the overall spatial distribution or to the mass segregation, the evolution of the Hubble model following collapse is slower than the one of the cold uniform model. The hubble system inherits segregation from its clumps and form a shallower, more stable model, while initially ejecting less stars.

\section{Conclusion}

We demonstrated a new method to create substructured initial conditions for nbody simulations of young stellar clusters. By applying a Hubble velocity field to an uniform model, we allow continuous fragmentation which stems from pre-existing (Poissonian) density fluctuations. The result is a fully self-consistent, subvirial and fragmented model. By studying clumps individuallys, we showed that they display a primordial mass segregation. This can be explained by a clump formation process in which massive stars act as seeds for substructure growth. This is confirmed by the mass function of clump members, clearly skewed towards massive stars. We find a good agreement between the outcome of our method, which is pure Nbody computations, and the characteristics of more computationnaly intensive hydrodynamical simulations of forming star clusters. When left to relax towards equilibrium, the Hubble-fragmented model undergoes a softer collapse than a cold uniform sphere: the virial equilibrium is reached more quickly and the internal structure evolves much more slowly over time. Having inherited segregation from its clumps, the Hubble model is more segregated than the uniform model after collapse.

The main idea of the Hubble fragmentation model is a new one in the context of stellar clusters and several things remain to be investigated, such as the fate of binaries population in such system, the influence of stellar evolution or the effect of a background galactic potential on the dynamical evolution of the cluster. These will be the object of upcoming contributions. Details on this new method and the analysis can be found in our paper, Dorval et al. (2015, MN submitted).

\section{References}

Allison, R. J., Goodwin, S. P., Parker, R. J., Portegies Zwart, S. F., de Grijs, R., \& Kouwenhoven, M. B. N. 2009, MNRAS, 395, 1449

André, P., Belloche, A., Motte, F., \& Peretto, N. 2007, A\& A, 472, 519

Caputo, D. P., de Vries, N., \& Portegies Zwart, S. 2014, MNRAS, 445, 674

Dorval, J., Boily, C. M., Moraux, \& E., Maschberger, T., \& Becker, C 2015, MNRAS, submitted, $\mathrm{MN}-15-2591-\mathrm{MJ}$

Goodwin, S. P., \& Whitworth, A. P. 2004, A\& A, 413, 929

Hénon, M. H. 1973, A\&A, 24, 229

Kuhn, M. A., Feigelson, E. D., Getman, K. V., Baddeley, A. J., Broos, P. S., Sills, A., Bate, M. R., Povich, M. S., Luhman, K. L., Busk, H. A., Naylor, T., \& King, R. R. 2014, ApJ, 631,976

Maschberger, T., Clarke, C. J., Bonnell, I. A., \& Kroupa, P. 2010, MNRAS, 404, 1061

McMillan, S. L. W., Vesperini, E., \& Portegies Zwart, S. F. 2007, Apj, 655, L45 